Journal of Applied AnALysis

Vol. 12, No. 2 (2006), pp. 283-292

\title{
ON MEASURABLE SIERPIŃSKI-ZYGMUND FUNCTIONS
}

\author{
A. B. KHARAZISHVILI \\ Received September 19, 2005 and, in revised form, January 27, 2006
}

\begin{abstract}
It is proved that there exists a Sierpiński-Zygmund function, which is measurable with respect to a certain invariant extension of the Lebesgue measure on the real line $\mathbb{R}$.
\end{abstract}

Let $E$ be a nonempty set and let $f: E \rightarrow \mathbb{R}$ be a function.

We say that $f$ is absolutely nonmeasurable if $f$ is nonmeasurable with respect to any nonzero $\sigma$-finite diffused (i.e., continuous) measure $\mu$ defined on a $\sigma$-algebra of subsets of $E$.

Recall that a set $X \subset \mathbb{R}$ is universal measure zero if, for every $\sigma$-finite diffused Borel measure $\mu$ on $\mathbb{R}$, the equality $\mu^{*}(X)=0$ is satisfied, where $\mu^{*}$ denotes, as usual, the outer measure associated with $\mu$.

It is well known that there exist uncountable universal measure zero subsets of $\mathbb{R}$ (see, e.g., [8]).

We have a characterization of absolutely nonmeasurable functions in terms of universal measure zero sets and preimages of singletons.

Theorem 1. Let $f: E \rightarrow \mathbb{R}$ be a function. The following two assertions are equivalent:

2000 Mathematics Subject Classification. Primary: 28A05, 28D05.

Key words and phrases. Sierpiński-Zygmund function, absolutely nonmeasurable function, universal measure zero set, extension of measure, thick graph, invariant measure.

ISSN 1425-6908（C) Heldermann Verlag. 
1) $f$ is absolutely nonmeasurable;

2) the range of $f$ is a universal measure zero subset of $\mathbb{R}$ and $\operatorname{card}\left(f^{-1}(t)\right) \leq \omega$ for each point $t \in \mathbb{R}$.

For the proof of this statement, see [5].

Let $\mathbf{c}$ denote the cardinality of the continuum. We would like to mention the following two straightforward consequences of Theorem 1.

I. If $\operatorname{card}(E)>\mathbf{c}$, then there are no absolutely nonmeasurable functions $f: E \rightarrow \mathbb{R}$

II. If the cardinality of any universal measure zero subset of $\mathbb{R}$ is strictly less than c, then there are no absolutely nonmeasurable functions $f: \mathbb{R} \rightarrow \mathbb{R}$.

The latter consequence shows us that absolutely nonmeasurable functions $f: \mathbb{R} \rightarrow \mathbb{R}$ exist if and only if there exists a universal measure zero set $X \subset \mathbb{R}$ with $\operatorname{card}(X)=$ c. More generally, absolutely nonmeasurable functions $f: E \rightarrow \mathbb{R}$ exist if and only if there exists a universal measure zero subset $X$ of $\mathbb{R}$ with $\operatorname{card}(X)=\operatorname{card}(E)$.

This also implies that the existence of an absolutely nonmeasurable function acting from $\mathbb{R}$ into $\mathbb{R}$ cannot be established within the theory ZFC. Indeed, there are models of set theory in which the negation of the Continuum Hypothesis holds and the cardinality of each universal measure zero subset of $\mathbb{R}$ does not exceed the first uncountable cardinal $\omega_{1}$ (see, e.g., [8]). Clearly, in such a model we do not have absolutely nonmeasurable functions acting from $\mathbb{R}$ into $\mathbb{R}$.

At the same time, by assuming Martin's Axiom and applying some properties of so-called generalized Luzin subsets of $\mathbb{R}$, it can be proved that there are injective additive absolutely nonmeasurable functions acting from $\mathbb{R}$ into $\mathbb{R}$. In other words, under Martin's Axiom, there exist absolutely nonmeasurable solutions of the Cauchy functional equation (see, for example, $[5])$.

Sierpiński and Zygmund constructed in their classical work [11] a function $f: \mathbb{R} \rightarrow \mathbb{R}$ having the following property: for each subset $Y$ of $\mathbb{R}$ with $\operatorname{card}(Y)=\mathbf{c}$, the restriction $f \mid Y$ is not continuous on $Y$.

The above-mentioned result of Sierpiński and Zygmund was essentially motivated by the theorem of Blumberg [1] stating that, for any function $g: \mathbb{R} \rightarrow \mathbb{R}$, there exists an everywhere dense subset $D$ of $\mathbb{R}$ such that the restriction $g \mid D$ is continuous on $D$. Obviously, the set $D$ being dense in $\mathbb{R}$ is infinite. The existence of a Sierpiński-Zygmund function shows that one cannot assert the uncountability of $D$.

There are many works devoted to Sierpiński-Zygmund functions. In those works various constructions are presented which yield further interesting 
examples of Sierpiński-Zygmund functions with additional properties important from the viewpoint of real analysis (see, e.g., [9] and references therein).

It is not difficult to prove that every Sierpiński-Zygmund function $f$ turns out to be nonmeasurable with respect to the completion of any nonzero $\sigma$ finite diffused Borel measure on $\mathbb{R}$ (see, for instance, [4, Chapter 6]). At the same time, we cannot assert that $f$ is absolutely nonmeasurable because it may happen (in those models of set theory where the Continuum Hypothesis fails to be true) that $\mathbf{c}>\operatorname{card}\left(f^{-1}(t)\right)>\omega$ for some point $t \in \mathbb{R}$ and, by virtue of Theorem 1 , in this case $f$ will be measurable with respect to a certain nonzero $\sigma$-finite diffused measure on $\mathbb{R}$.

The present paper is devoted to related measurability properties of Sierpiński-Zygmund functions. The main goal of the paper is to demonstrate that there are Sierpiński-Zygmund functions measurable with respect to some invariant extensions of the standard Lebesgue measure $\lambda$ on $\mathbb{R}$.

For this purpose, we need several auxiliary propositions.

Lemma 1. Let $f: \mathbb{R} \rightarrow \mathbb{R}$ be a function satisfying the following condition: for each subset $Y$ of $\mathbb{R}$ with $\operatorname{card}(Y)=\mathbf{c}$, the restriction $f \mid Y$ is not a Borel mapping. Then $f$ is a Sierpinski-Zygmund function.

This lemma is trivial. However, it plays an essential role for our considerations below. In this context, let us point out that in Chapter 5 of [4] a construction of a function $f$ satisfying the above-mention condition is discussed in detail.

As usual, we denote by $\mathbb{T}$ the one-dimensional unit torus in the Euclidean plane $\mathbb{R}^{2}$. Recall that $\mathbb{T}$ is a commutative compact topological group canonically isomorphic to the group of all rotations of the plane $\mathbb{R}^{2}$ about its origin.

Lemma 2. Let $\phi: \mathbb{R} \rightarrow \mathbb{T}$ be the canonical surjective continuous group homomorphism defined by

$$
\phi(x)=(\cos x, \sin x) \quad(x \in \mathbb{R}) .
$$

There exists a mapping $\psi: \mathbb{T} \rightarrow \mathbb{R}$ such that:

1) $\phi \circ \psi$ coincides with the identity transformation of $\mathbb{T}$;

2) $\psi$ is discontinuous only at one point of $\mathbb{T}$ (hence, $\psi$ is a Borel mapping).

We omit an easy proof of Lemma 2 (actually, this simple lemma is a very particular case of the theorem of Kuratowski and Ryll-Nardzewski [7] on measurable selectors). 
The torus $\mathbb{T}$ being a compact topological group carries the Haar probability measure $\nu$. In fact, the completion of $\nu$ coincides with the Lebesgue probability measure on $\mathbb{T}$ invariant under all translations of $\mathbb{T}$.

As usual, we denote by $\lambda \times \nu$ the product measure whose multipliers are $\lambda$ and $\nu$. For our further purposes, it will be convenient to denote by the same symbol $\lambda \times \nu$ the completion of the above-mentioned product measure.

We say that a function $f: \mathbb{R} \rightarrow \mathbb{T}$ has thick graph if every $(\lambda \times \nu)$ measurable set $Z$ with $(\lambda \times \nu)(Z)>0$ intersects the graph of $f$.

Lemma 3. Let $f: \mathbb{R} \rightarrow \mathbb{T}$ be a group homomorphism whose graph is thick. For any set $Z \in \operatorname{dom}(\lambda \times \nu)$, let us put

$$
Z^{\prime}=\{x \in \mathbb{R}:(x, f(x)) \in Z\}
$$

and define the class of sets

$$
\mathcal{S}=\left\{Z^{\prime}: Z \in \operatorname{dom}(\lambda \times \nu)\right\} .
$$

Finally, define a functional $\mu$ on $\mathcal{S}$ by the formula

$$
\mu\left(Z^{\prime}\right)=(\lambda \times \nu)(Z) \quad\left(Z^{\prime} \in \mathcal{S}\right) .
$$

Then the following relations hold:

1) $\mathcal{S}$ is a $\sigma$-algebra of subsets of $\mathbb{R}$ containing $\operatorname{dom}(\lambda)$ and invariant under the group of all isometric transformations of $\mathbb{R}$;

2) the functional $\mu$ is well-defined;

3) $\mu$ is a measure on $\mathcal{S}$ extending $\lambda$ and invariant under the same group of all isometries of $\mathbb{R}$;

4) the original homomorphism $f$ is measurable with respect to $\mu$.

Proof. We use the argument given in [6] (cf. also [3]).

If $Z \in \operatorname{dom}(\lambda \times \nu)$ and $Z_{i} \in \operatorname{dom}(\lambda \times \nu)$ for each index $i$ from a countable set $I$, then we can write

$$
\begin{gathered}
\mathbb{R} \backslash Z^{\prime}=\left\{x \in \mathbb{R}:(x, f(x)) \in \mathbb{R}^{2} \backslash Z\right\}, \\
\bigcup\left\{Z_{i}^{\prime}: i \in I\right\}=\left\{x \in \mathbb{R}:(x, f(x)) \in \bigcup\left\{Z_{i}: i \in I\right\}\right\},
\end{gathered}
$$

which shows us that $\mathcal{S}$ is a $\sigma$-algebra of subsets of $\mathbb{R}$.

The invariance of $\mathcal{S}$ under the group of all isometries of $\mathbb{R}$ follows directly from the invariance of $\operatorname{dom}(\lambda \times \nu)$ under all translations of the product group $\mathbb{R} \times \mathbb{T}$ and under the symmetry of this group.

If, for a set $Z^{\prime} \in \mathcal{S}$, we have simultaneously $Z^{\prime}=\left\{x \in \mathbb{R}:(x, f(x)) \in Z_{1}\right\}$ and $Z^{\prime}=\left\{x \in \mathbb{R}:(x, f(x)) \in Z_{2}\right\}$, where $Z_{1}$ and $Z_{2}$ are some $(\lambda \times \nu)$ measurable sets, then

$$
\left\{x \in \mathbb{R}:(x, f(x)) \in Z_{1} \triangle Z_{2}\right\}=\emptyset .
$$


In view of the thickness of the graph of $f$, we claim that $(\lambda \times \nu)\left(Z_{1} \triangle Z_{2}\right)=0$, which also implies that $(\lambda \times \nu)\left(Z_{1}\right)=(\lambda \times \nu)\left(Z_{2}\right)$. This establishes the correctness of the definition of $\mu$.

In a similar way, we prove the countable additivity of $\mu$ and its invariance under the group of all isometries of $\mathbb{R}$.

Further, if $X$ is an arbitrary $\lambda$-measurable set, then we can write

$X=\{x \in \mathbb{R}:(x, f(x)) \in X \times \mathbb{T}\}, \quad X \in \mathcal{S}, \quad \mu(X)=(\lambda \times \nu)(X \times \mathbb{T})=\lambda(X)$

and, therefore, the measure $\mu$ extends $\lambda$.

Finally, for any Borel set $B \subset \mathbb{T}$, we have

$f^{-1}(B)=\{x \in \mathbb{R}:(x, f(x)) \in \mathbb{R} \times B\}, \quad \mathbb{R} \times B \in \operatorname{dom}(\lambda \times \nu), \quad f^{-1}(B) \in \mathcal{S}$,

which shows that $f$ is measurable with respect to $\mu$.

This completes the proof of Lemma 3.

Lemma 4. Let $(G,+)$ be an infinite commutative divisible group such that, for any nonzero natural number $n$ and for any element $g \in G$, the equation $n x=g$ has at most countably many solutions in $G$. Let $A$ be an arbitrary subset of $G$. There exists a subgroup $[A]$ of $G$ satisfying the following conditions:

1) $A \subset[A]$;

2) $\operatorname{card}([A])=\operatorname{card}(A)+\omega$;

3) for each nonzero natural number $n$ and for each element $g \in[A]$, all solutions of the equation $n x=g$ belong to $[A]$ (in particular, $[A]$ is a divisible subgroup of $G$ ).

Proof. Construct by recursion a sequence $\left(G_{0}, G_{1}, \ldots, G_{k}, \ldots\right)$ of subgroups of $G$. First of all, put:

$G_{0}=$ the group generated by $A$.

Suppose that the subgroup $G_{k}$ has already been defined satisfying the equality $\operatorname{card}\left(G_{k}\right)=\operatorname{card}(A)+\omega$. Denote by $X_{k}$ the set of all solutions of the equations

$$
n x=g \quad\left(n=1,2, \ldots ; g \in G_{k}\right) .
$$

Taking into account the assumption of the lemma, we get

$$
\operatorname{card}\left(X_{k}\right)=\operatorname{card}\left(G_{k}\right)+\omega=\operatorname{card}(A)+\omega .
$$

Let us put:

$G_{k+1}=$ the group generated by the set $G_{k} \cup X_{k}$.

Proceeding in this way, we obtain the increasing sequence $\left(G_{0}, G_{1}, \ldots, G_{k}, \ldots\right)$ of subgroups of $G$.

Finally, denote $[A]=\bigcup\left\{G_{k}: k<\omega\right\}$. A straightforward verification shows that the group $[A]$ is the required one. 
Remark 1. Lemma 4 has a direct analog for infinite noncommutative divisible groups $(G, \cdot)$.

Lemma 5. There exists a group homomorphism

$$
f: \mathbb{R} \rightarrow \mathbb{T}
$$

possessing the following properties:

1) the graph of $f$ is thick;

2) for any uncountable Borel subset $Y$ of $\mathbb{R}$ and for any Borel mapping $h: Y \rightarrow \mathbb{T}$, we have the inequality

$$
\operatorname{card}(\{x \in \mathbb{R}: f(x)=h(x)\})<\mathbf{c} .
$$

Proof. We shall construct the required homomorphism $f$ by using the method of transfinite recursion.

Let $\alpha$ denote the least ordinal number of cardinality $\mathbf{c}$.

Let $\preceq$ be a well-ordering of $\mathbb{R}$ isomorphic to $\alpha$.

Let $\left\{h_{\xi}: \xi<\alpha\right\}$ be an enumeration of all Borel mappings acting from uncountable Borel subsets of $\mathbb{R}$ into $\mathbb{T}$.

Let $\left\{Z_{\xi}: \xi<\alpha\right\}$ be an enumeration of all those Borel subsets of $\mathbb{R} \times \mathbb{T}$ whose $(\lambda \times \nu)$-measure is strictly positive. We may assume, without loss of generality, that the range of the partial family

$$
\left\{Z_{\xi}: \xi<\alpha, \xi \text { is an odd ordinal }\right\}
$$

coincides with the range of the entire family $\left\{Z_{\xi}: \xi<\alpha\right\}$.

Under this notation, we are going to define four $\alpha$-sequences

$$
\begin{aligned}
& \left\{x_{\xi}: \xi<\alpha\right\}, \quad\left\{y_{\xi}: \xi<\alpha\right\}, \\
& \left\{V_{\xi}: \xi<\alpha\right\}, \quad\left\{f_{\xi}: \xi<\alpha\right\}
\end{aligned}
$$

satisfying the following relations:

(a) $\left\{x_{\xi}: \xi<\alpha\right\}$ is a Hamel basis of $\mathbb{R}$;

(b) for each ordinal $\xi<\alpha$, the set $V_{\xi}$ is the vector subspace of $\mathbb{R}$, over the field $\mathbb{Q}$ of all rationals, generated by $\left\{x_{\zeta}: \zeta \leq \xi\right\}$;

(c) for each ordinal $\xi<\alpha$, we have the group homomorphism $f_{\xi}: V_{\xi} \rightarrow \mathbb{T}$ such that $f_{\xi}\left(x_{\xi}\right)=y_{\xi}$

(d) if $\zeta<\xi<\alpha$, then $f_{\xi}$ extends $f_{\zeta}$;

(e) for each odd ordinal $\xi<\alpha$, we have $\left(x_{\xi}, y_{\xi}\right) \in Z_{\xi}$;

(f) if $\xi<\alpha$, then we have

$$
f_{\xi}\left(q x_{\xi}+v\right) \neq h_{\zeta}\left(q x_{\xi}+v\right)
$$

for all $\zeta<\xi, q \in \mathbb{Q} \backslash\{0\}, v \in \bigcup\left\{V_{\zeta}: \zeta<\xi\right\}, q x_{\xi}+v \in \operatorname{dom}\left(h_{\zeta}\right)$. 
Suppose that, for an ordinal $\xi<\alpha$, the partial $\xi$-sequences

$$
\begin{array}{ll}
\left\{x_{\zeta}: \zeta<\xi\right\}, & \left\{y_{\zeta}: \zeta<\xi\right\}, \\
\left\{V_{\zeta}: \zeta<\xi\right\}, & \left\{f_{\zeta}: \zeta<\xi\right\}
\end{array}
$$

have already been constructed. Let us put:

$$
V^{\prime}=\bigcup\left\{V_{\zeta}: \zeta<\xi\right\}, \quad f^{\prime}=\bigcup\left\{f_{\zeta}: \zeta<\xi\right\} .
$$

Applying (d), we claim that $f^{\prime}$ is a group homomorphism from $V^{\prime}$ into $\mathbb{T}$. Now, consider two possible cases.

1. The ordinal $\xi$ is even. In this case, let $x$ be the least element (with respect to $\preceq$ ) of $\mathbb{R} \backslash V^{\prime}$ and let

$$
A=\left\{h_{\zeta}(q x+v): \zeta<\xi, q \in \mathbb{Q} \backslash\{0\}, v \in V^{\prime}, q x+v \in \operatorname{dom}\left(h_{\zeta}\right)\right\}+f^{\prime}\left(V^{\prime}\right) .
$$

According to Lemma 4, we have

$$
\operatorname{card}([A])=\operatorname{card}(A)+\omega \leq \operatorname{card}(\xi)+\omega<\mathbf{c} .
$$

Choose an element $y \in \mathbb{T} \backslash[A]$ and put $x_{\xi}=x, y_{\xi}=y$. Taking into account the fact that $\mathbb{T}$ is a divisible group, we can extend $f^{\prime}$ to a group homomorphism

$$
f_{\xi}: V_{\xi} \rightarrow \mathbb{T}
$$

satisfying the condition $f_{\xi}\left(x_{\xi}\right)=y_{\xi}$.

2. The ordinal $\xi$ is odd. In this case, we apply the classical Fubini theorem to the set $Z_{\xi}$ and choose an element $x \in \mathbb{R} \backslash V^{\prime}$ such that

$$
\nu\left(\left\{t \in \mathbb{T}:(x, t) \in Z_{\xi}\right\}\right)>0 .
$$

Further, we choose an element

$$
y \in\left\{t \in \mathbb{T}:(x, t) \in Z_{\xi}\right\} \backslash[A]
$$

(where $A$ is defined as in the case 1 ) and put again $x_{\xi}=x, y_{\xi}=y$. Obviously, we have $\left(x_{\xi}, y_{\xi}\right) \in Z_{\xi}$. As above, we define a group homomorphism $f_{\xi}: V_{\xi} \rightarrow \mathbb{T}$ extending $f^{\prime}$ and satisfying the condition $f_{\xi}\left(x_{\xi}\right)=y_{\xi}$.

Proceeding in this manner, we will be able to construct the required $\alpha$-sequences. Now, we put

$$
f=\bigcup\left\{f_{\xi}: \xi<\alpha\right\}
$$

In view of (a), this $f$ is a group homomorphism from $\mathbb{R}$ into $\mathbb{T}$.

In view of (e), the graph of $f$ is thick in the product space $\mathbb{R} \times \mathbb{T}$.

Finally, relation (f) implies that the inequality

$$
\operatorname{card}\left(\left\{x \in \mathbb{R}: f(x)=h_{\xi}(x)\right\}\right)<\mathbf{c}
$$

holds for all ordinals $\xi<\alpha$. This also yields that, for any set $X \subset \mathbb{R}$ with $\operatorname{card}(X)=\mathbf{c}$, the restriction of $f$ to $X$ is not a Borel mapping. To show the last fact, suppose otherwise, i.e., suppose that $f \mid X$ is Borel. Then, 
according to a well-known statement of classical descriptive set theory (see, e.g., [4, Chapter 5]), the function $f \mid X$ can be extended to a $\mathbb{T}$-valued Borel function $f^{*}$ defined on a Borel subset of $\mathbb{R}$. Since $\operatorname{card}\left(\operatorname{dom}\left(f^{*}\right)\right)=\mathbf{c}$, we claim that $f^{*}=h_{\xi}$ for some ordinal $\xi<\alpha$. But this is impossible in view of the above inequality.

Lemma 5 has thus been proved.

Theorem 2. There exists a Sierpiński-Zygmund function

$$
\chi: \mathbb{R} \rightarrow \mathbb{R}
$$

measurable with respect to some invariant extension of Lebesgue measure $\lambda$.

Proof. Let $f$ be as in Lemma 5. By virtue of Lemma 3, $f$ is measurable with respect to a certain measure $\mu$ on $\mathbb{R}$ which extends $\lambda$ and is invariant under the group of all isometries of $\mathbb{R}$. Let us put

$$
\chi=\psi \circ f,
$$

where $\psi: \mathbb{T} \rightarrow \mathbb{R}$ denotes the Borel mapping of Lemma 2 .

We assert that $\chi$ is the required Sierpiński-Zygmund function.

Indeed, $\chi$ is a $\mu$-measurable function as the composition of two functions, first of which is $\mu$-measurable and the second one is Borel.

Let $X$ be a subset of $\mathbb{R}$ with $\operatorname{card}(X)=\mathbf{c}$. It suffices to show that the restriction $\chi \mid X$ is not a Borel mapping. Suppose otherwise, i.e., suppose that $\chi \mid X$ is Borel. Then

$$
\phi \circ \chi|X=\phi \circ \psi \circ f| X=f \mid X
$$

must be Borel, too, which is impossible in view of Lemma 5.

The contradiction obtained ends the proof of our theorem.

Remark 2. We cannot assert that the function $\chi$ of Theorem 1 is additive. In this context, the following question seems to be interesting: does there exist an additive Sierpiński-Zygmund function acting from $\mathbb{R}$ into $\mathbb{R}$ and measurable with respect to some invariant extension of $\lambda$ ?

Note that, by applying the method described above, it is possible to prove that there exists an additive Sierpiński-Zygmund function acting from $\mathbb{R}$ into $\mathbb{R}$ and measurable with respect to some quasiinvariant extension of $\lambda$.

Replacing $\mathbb{R}$ by a Polish topological vector space equipped with a nonzero $\sigma$-finite diffused Borel measure, we come to a certain analog of Theorem 2. 
Theorem 3. Let $E \neq\{0\}$ be a Polish topological vector space and let $\mu$ be a nonzero $\sigma$-finite diffused Borel measure on $E$. There exist a measure $\mu^{\prime}$ on $E$ extending $\mu$ and a Sierpinski-Zygmund function $\chi: E \rightarrow \mathbb{R}$ measurable with respect to $\mu^{\prime}$. Moreover, if $\mu$ is invariant (quasiinvariant) under a group $G \subset E$, then $\mu^{\prime}$ can also be chosen invariant (quasiinvariant) under the same $G$.

The proof of this statement is similar to the proof of Theorem 2 .

Remark 3. It is well known that if $E$ is an infinite-dimensional Polish topological vector space, then there exists no nonzero $\sigma$-finite Borel measure on $E$ quasiinvariant under the group of all translations of $E$. On the other hand, for some $E$, it is possible to construct a nonzero $\sigma$-finite Borel measure on $E$ invariant under an everywhere dense vector subspace of $E$ (see, e.g., $[2])$.

Remark 4. Let $\chi: \mathbb{R} \rightarrow \mathbb{R}$ be an arbitrary Sierpiński-Zygmund function. By assuming that all sets in $\mathbb{R}$ of cardinality strictly less than $\mathbf{c}$ are of first category, it is not difficult to show that, for any second category set $X \subset \mathbb{R}$, the restriction $\chi \mid X$ cannot be extended to a function $\chi^{*}: \mathbb{R} \rightarrow \mathbb{R}$ possessing the Baire property.

Similarly, let $f: \mathbb{R} \rightarrow \mathbb{R}$ be a Sierpiński-Zygmund function. By assuming that all sets in $\mathbb{R}$ of cardinality strictly less than $\mathbf{c}$ are of Lebesgue measure zero, it is not hard to prove that, for any set $X \subset \mathbb{R}$ of strictly positive outer Lebesgue measure, the restriction $f \mid X$ cannot be extended to a function $f^{*}: \mathbb{R} \rightarrow \mathbb{R}$ measurable in the Lebesgue sense.

In this context, we would like to recall that the Baire property is a certain topological analog of measurability (see, for instance, [10]).

\section{References}

[1] Blumberg, H., New properties of all real functions, Trans. Amer. Math. Soc. 24 (1922), 113-128.

[2] Kharazishvili, A. B., Invariant measures in Hilbert space (in Russian), Soobshch. Akad. Nauk Gruzin. SSR 114(1) 1984, 41-44.

[3] Kharazishvili, A. B., Transformation Groups and Invariant Masures. Set-Theoretical Aspects. World Scientific Publishing Co., Inc., River Edge, NJ, 1998.

[4] Kharazishvili, A. B., Strange Functions in Real Analysis, Pure and Appl. Math. (N. Y.) 229, Marcel Dekker, Inc., New York, 2000.

[5] Kharazishvili, A. B., Kirtadze, A., On the measurability of functions with respect to certain classes of measures, Georgian Math. J. 11(3) (2004), 489-494.

[6] Kodaira, K., Kakutani, S., A nonseparable translation-invariant extension of the Lebesgue measure space. Ann. of Math. (2) 52 (1950), 574-579. 
[7] Kuratowski, K., Ryll-Nardzewski, Cz., A general theorem on selectors, Bull. Acad. Polon. Sci. Sér. Sci. Math. Astronom. Phys. 13(6) (1965), 397-402.

[8] Miller, A. W., Special subsets of the real line, in "Handbook of Set-Theoretic Topology", North-Holland, Amsterdam, 1984.

[9] Natkaniec, T., Rosen, H., An example of an additive almost continuous SierpińskiZygmund function, Real Anal. Exchange 30(1) (2004-2005), 261-266.

[10] J.C. Oxtoby, Measure and Category, Springer-Verlag, Berlin, 1971.

[11] Sierpiński, W., Zygmund, A., Sur une fonction qui est discontinue sur tout ensemble de puissance du continu, Fund. Math. 4 (1923), 316-318.

\author{
A. B. KharaZishViLI \\ I.Vekua Institute of Applied Mathematics \\ of Tbilisi State University \\ University Street 2 \\ TbiLisi 0143, Georgia \\ AND \\ A. Razmadze Institute of Mathematics \\ of Georgian Academy of Sciences \\ M. Alexidze Street 1 \\ Tbilisi 0194, Georgia \\ E-MAIL: KHARAZ2@YAHOO.COM
}

